\begin{tabular}{|c|c|}
\hline Title & Formation of GaN-Porous Structures U sing Photo-A ssisted Electrochemical Process in Back-Side Illumination Mode \\
\hline Author(s) & W atanabe, A kio; Kumazaki, Y usuke; Y atabe, Zenji; Sato, Taketomo \\
\hline Citation & $\begin{array}{l}\text { ECS Electrochemistry Letters, 4(5), H11-H13 } \\
\text { https://doi.org/10.1149/2.0031505eel }\end{array}$ \\
\hline Issue Date & 2015-03-14 \\
\hline Doc URL & http:/hdl. handle.net/2115/58546 \\
\hline Rights & $\begin{array}{l}\text { (1) The Electrochemical Society, Inc. 2015. All rights reserved. Except as provided under U.S. copyright law, this work } \\
\text { may not be reproduced, resold, distributed, or modified without the express permission of The Electrochemical Society } \\
\text { (ECS). The archival version of this work was published in ECS Electrochemistry Letters. }\end{array}$ \\
\hline Type & article \\
\hline File Information & H11.full.pdf \\
\hline
\end{tabular}

Instructions for use 


\title{
Formation of GaN-Porous Structures Using Photo-Assisted Electrochemical Process in Back-Side Illumination Mode
}

\author{
Akio Watanabe, ${ }^{\mathrm{Z}}$ Yusuke Kumazaki, Zenji Yatabe, and Taketomo Sato* \\ Research Center for Integrated Quantum Electronics (RCIQE), Hokkaido University, Sapporo 060-8628, Japan
}

\begin{abstract}
We investigated the structural features of gallium-nitride-porous structures formed using the photo-assisted electrochemical process in the back-side illumination (BSI) mode. The pore diameter and depth were strongly affected by the direction of illumination, where higher controllability was achieved compared with front-side illumination. The spectroscopic measurements revealed that illumination with photon energy below the bulk bandgap plays an important role in pore formation. We propose a formation model by considering the Franz-Keldysh effect that can consistently explain the obtained experimental data in which anodic etching occurs only at the pore tips under the high electric field induced in the depletion region.

(C) 2015 The Electrochemical Society. [DOI: 10.1149/2.0031505eel] All rights reserved.
\end{abstract}

Manuscript submitted December 26, 2014; revised manuscript received February 5, 2015. Published March 14, 2015.

Gallium nitride $(\mathrm{GaN})$ is a III/V direct bandgap semiconductor that exhibits high-thermal, chemical, and mechanical stability and is relatively harmless to humans and the environment because it does not contain toxic substances such as arsenic (As). Most notably, GaN is a wide-bandgap material with $3.4 \mathrm{eV}$ of energy, which can be varied from 0.65 to $6.0 \mathrm{eV}$ by alloying it with indium nitride $(\mathrm{InN})$ and aluminum nitride (AlN). On the basis of these excellent properties, various functional devices have been designed, for example ultraviolet (UV) laser diodes, ${ }^{1}$ chemical sensors, ${ }^{2}$ and photo-electrodes for water splitting. ${ }^{3}$ The well known challenge to improve these device's performance is to use nanostructures in which the unique optical and electrical properties, such as quantum effects and increased surface sensitivity, appear with a large surface area to volume ratio.

Electrochemically formed porous structures, which have been applied for various materials, ${ }^{4-7}$ are promising nanostructures for the above-mentioned applications. Many researchers have reported the electrochemical formation of GaN-porous structures. ${ }^{8-10}$ However, their formation mechanism has not been fully understood. One of the reasons is that the photo-assisted electrochemical process is commonly used in which the formation process becomes more complicated due to the supply of photo-carries generated by illumination. We previously argued that it is difficult with front-side illumination (FSI) to control the structural properties of GaN-porous structures. ${ }^{11}$ We believe that the optimization of the supply of photo-carriers is one of the key issues for controlling of the structural properties.

In this study, we formed GaN-porous structures using the photoassisted electrochemical process in the back-side illumination (BSI) mode, for the first time, and compared with it that in FSI mode. From both experimental and theoretical aspects, we discuss the formation mechanism of GaN-porous structures in BSI mode.

The electrochemical setup used in this study is schematically shown in Figure 1. A custom-made cell equipped with a crystal window and Indium Tin Oxide (ITO) plate was used for both the photo-assisted electrochemical process and spectroscopic measurements. The electrochemical cell has three electrodes, i.e., an n-type GaN electrode as a working electrode (W.E.), Pt counter electrode (C.E.), and $\mathrm{Ag} / \mathrm{AgCl}$ reference electrode (R.E.). We used $\mathrm{GaN}$ epitaxial layers grown on freestanding $\mathrm{GaN}$ substrates or sapphire substrates as the W.E. The electrolyte consists of $1 \mathrm{~mol} / \mathrm{L} \mathrm{H}_{2} \mathrm{SO}_{4}$ and $1 \mathrm{~mol} / \mathrm{L}$ $\mathrm{H}_{3} \mathrm{PO}_{4}(\mathrm{pH}=2.5)$.

The GaN-porous structures were formed using the photo-assisted electrochemical process both in the FSI and BSI modes using a xenon (Xe) lamp as a UV light source, as shown in Fig. 1. The photogenerated holes, $\mathrm{h}^{+}$, are used at the electrolyte/n-GaN interface for the following anodic reaction. ${ }^{12}$

$$
2 \mathrm{GaN}+6 \mathrm{~h}^{+} \rightarrow 2 \mathrm{Ga}^{3+}+\mathrm{N}_{2}
$$

Various electrochemical conditions such as applied voltage $V_{\mathrm{a}}$, UVintensity $P_{\mathrm{IN}}$, and anodization time $t_{\mathrm{a}}$, were precisely controlled using a potentiostat connected to a computer system monitoring the anodic-reaction current density $J_{\mathrm{a}}$. The structural properties of the $\mathrm{GaN}$-porous samples formed under different conditions were compared using a scanning electron microscope (SEM) with a Hitachi SU-8010 system. Spectroscopic properties, such as photo-reflectance, transmittance, and absorbance, were evaluated using an HR4000CGUV-NIR spectrometer (Ocean Optics) under the voltage applied in the same setup shown in Fig. 1.

Figures $2 \mathrm{a}-2 \mathrm{~d}$ show top and cross-sectional SEM images of the GaN-porous samples formed after $t_{\mathrm{a}}=600$ and $1800 \mathrm{~s}$ by comparing between the FSI and BSI modes. The $V_{\mathrm{a}}$ and $P_{\mathrm{IN}}$ were adjusted by monitoring the $J_{\mathrm{a}}$ to be about the same between both modes. No anodic current and no porous structure was observed at the same bias condition in the dark. For the FSI mode shown in Figs. $2 \mathrm{a}$ and $2 \mathrm{~b}$, the pore diameter measured from top images increased with anodization time, whereas the pore depth $d_{\mathrm{p}}$ measured from the cross-section did not increase. On the other hand, $d_{\mathrm{p}}$ increased with anodization time in BSI mode, as shown in Figs. $2 \mathrm{c}$ and $2 \mathrm{~d}$, where the pore was the almost the same throughout all parts of the porous layer.

Figure 3 shows the relationship between $d_{\mathrm{p}}$ and the charge density $Q$ passing through the working electrode during the photo-assisted electrochemical process. In FSI mode, $d_{\mathrm{p}}$ increased with $Q$ until around $400 \mathrm{mC} / \mathrm{cm}^{2}\left(t_{\mathrm{a}}=1200 \mathrm{sec}\right)$, but it largely decreased after that. In BSI mode, however, $d_{\mathrm{p}}$ increased almost linearly with $Q$, showing no decrease in $Q$ over $1000 \mathrm{mC} / \mathrm{cm}^{2}$ in this study. These results come from the difference in the supply method of holes used for the anodization reaction. The FSI mode generates holes near the topsurface due to FSI. As $Q$ increased to more than about $400 \mathrm{mC} / \mathrm{cm}^{2}\left(t_{\mathrm{a}}\right.$ $=1200 \mathrm{sec}$ ), the pore wall thinned to breaking point and was removed from the top-surface. In BSI mode, on the other hand, the holes were

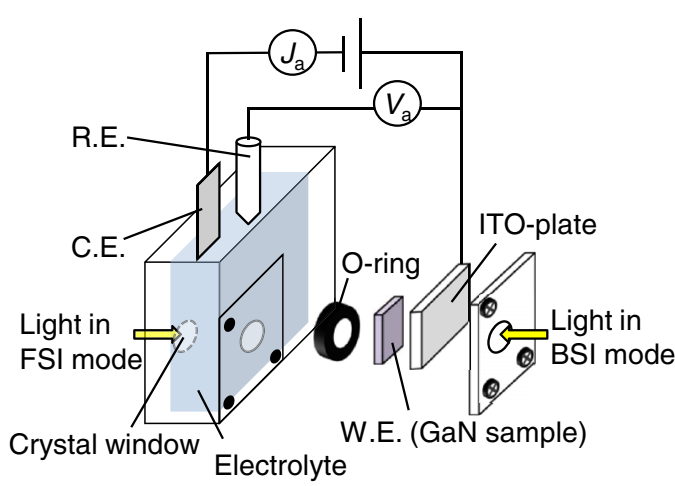

Figure 1. Schematic illustration of electrochemical setup used for both formation of porous structures and spectroscopic measurements. 


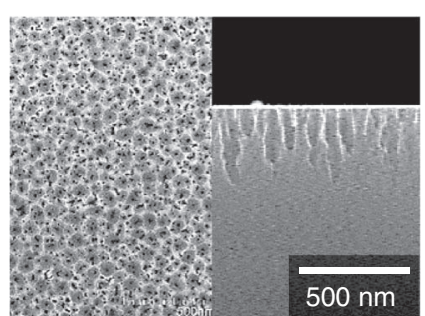

(a)

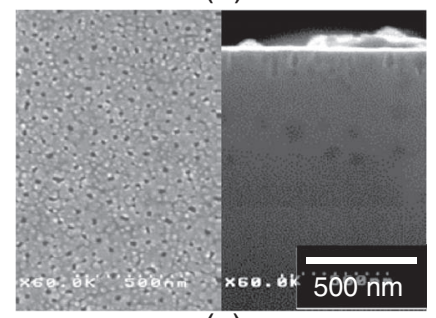

(c)

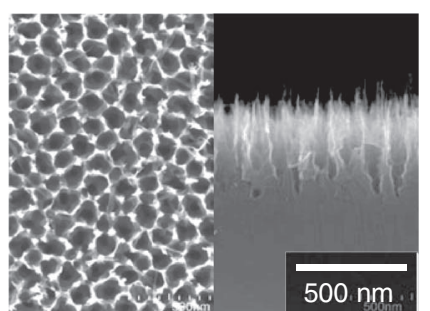

(b)

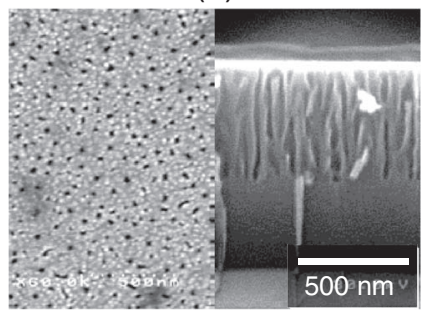

(d)
Figure 2. Top and cross-sectional SEM images of GaN-porous samples formed in FSI mode with $V_{\mathrm{a}}=1 \mathrm{~V}, P_{\mathrm{IN}}=5 \mathrm{~mW} / \mathrm{cm}^{2}$ and different $t_{\mathrm{a}}$ : (a) $t_{\mathrm{a}}=600 \mathrm{~s}$ and (b) $t_{\mathrm{a}}=1800 \mathrm{~s}$, and samples formed in BSI mode with $V_{\mathrm{a}}$ $=5 \mathrm{~V}, P_{\mathrm{IN}}=65 \mathrm{~mW} / \mathrm{cm}^{2}$ and different $t_{\mathrm{a}}:$ (c) $t_{\mathrm{a}}=600 \mathrm{~s}$ and (d) $t_{\mathrm{a}}=1800 \mathrm{~s}$.

supplied from the back surface or supplied only at the pore tips due to BSI. Since the anodic reaction occurred only at the pore tips, the pore was etched in the vertical direction, whose depth linearly increased with $Q$. From these results, we found that the BSI mode is very powerful for controlling the structural properties of GaN-porous structures such as pore diameter and $d_{\mathrm{p}}$ compared with the FSI mode.

The BSI mode was first reported on the formation of Si-porous structures. ${ }^{13}$ Light with photon energy $h v$ above $1.43 \mathrm{eV}$ was irradiated, whose energy was larger than the bandgap of Si. The holes generated at the back-surface diffused toward the pore tips to yield the anodic-reaction since the diffusion length of minority carriers in $\mathrm{Si}$ was long enough. However, the situation in BSI mode for $\mathrm{GaN}$ is quite different. Namely, the diffusion length of holes in n-type GaN is several hundred $\mathrm{nm}$ at most, which is much smaller than the sample thickness of $400 \mu \mathrm{m}$ used in this study. In such a situation, the holes generated near the back-surface cannot contribute to the anodic reaction because they recombine until reaching the pore tips.

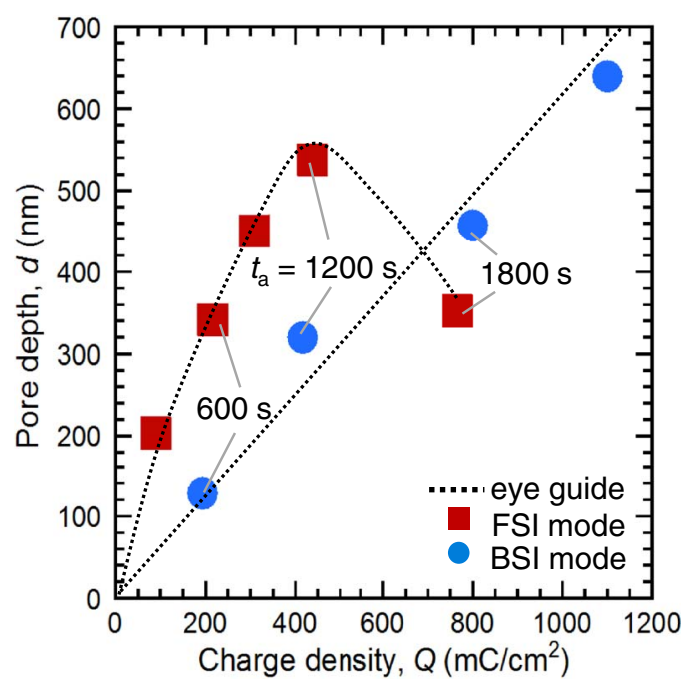

Figure 3. Relationship between pore depth $d_{\mathrm{p}}$ and charge density $Q$ passing through during photo-assisted electrochemical process.

\begin{tabular}{|c|c|c|}
\hline $\begin{array}{l}\text { Wavelength }(\mathrm{nm}) / \\
\text { Photon energy }(\mathrm{eV})\end{array}$ & $\begin{array}{c}\text { Anodic-reaction current } \\
\text { density } J_{\mathrm{a}}\left(\mathrm{A} / \mathrm{cm}^{2}\right)\end{array}$ & SEM observation \\
\hline $350 / 3.54$ & $<10^{-6}$ & No structure \\
\hline $360 / 3.44$ & $0.54 \sim 1.69 \times 10^{-5}$ & Shallow etching \\
\hline $370 / 3.35$ & $2.76 \sim 8.39 \times 10^{-4}$ & Porous formation \\
\hline $380 / 3.26$ & $0.44 \sim 2.37 \times 10^{-4}$ & Porous formation \\
\hline $390 / 3.18$ & $<10^{-6}$ & No structure \\
\hline $400 / 3.10$ & $<10^{-6}$ & No structure \\
\hline
\end{tabular}

To clarify the formation mechanism of GaN-porous structures in BSI mode, we conducted the photo-assisted electrochemical process by changing monochromatic-light illumination using an optical filter. Table I summarizes the anodic-reaction $J_{\mathrm{a}}$ and the results of SEM observation on whether the porous structure was formed. The full width at half maximum of the band-path filter was $10 \mathrm{~nm}$. From a series of experiments, we found that porous structures were formed on the GaN samples under monochromatic light with wavelengths of 370 and $380 \mathrm{~nm}$, whereas neither porous formation nor anodic etching was observed at wavelengths of 350,390 , and $400 \mathrm{~nm}$. It should be noted that the largest $J_{\mathrm{a}}$ was observed at a wavelength of $370 \mathrm{~nm}$, corresponding to $h v$ of $3.35 \mathrm{eV}$, which is smaller than the bandgap of $\mathrm{GaN}$. These results clearly indicate that the light with $h v$ below the bandgap contributes to the formation of $\mathrm{GaN}$-porous structures in BSI mode.

The Franz-Keldysh effect is one possible phenomenon to explain the present results. Franz ${ }^{14}$ and Keldysh ${ }^{15}$ pointed out that the high electric field may cause a red-shift of the absorption edge, leading to the presence of absorption below the bulk bandgap. Cavallini et al. reported that the Franz-Keldysh effect is applicable for GaN nanowires to argue that the photo-absorption properties depended strongly on the wire diameter. ${ }^{16}$ We believe that this effect is similarly applicable to the present electrochemical process, where a high electric field is induced in the depletion region formed at the interface between the electrolyte and n-type GaN. In the depletion region, the Franz-Keldysh relative absorption coefficient $\alpha$ is expressed as a function of both $h v$ and $V_{\mathrm{a}}$, as follows: ${ }^{17,18}$

$$
\alpha\left(h \nu, V_{a}\right)=\alpha_{0} \exp \left(-\left|\frac{h \nu-E_{g a p}}{\Delta E\left(V_{a}\right)}\right|^{\frac{3}{2}}\right),
$$

where $E_{\text {gap }}$ is the bulk bandgap ( $=3.44 \mathrm{eV}$ for $\mathrm{GaN}$ ) and $\alpha_{0}$ is the absorption coefficient at $h \nu=E_{\text {gap }}$. The term $\Delta E$ in Eq. 2 is the red-shift energy of the absorption edge and expressed by

$$
\Delta E\left(V_{a}\right)=\frac{2}{3} \frac{\left(q \hbar \xi\left(V_{a}\right)\right)^{\frac{2}{3}}}{\left(m^{*}\right)^{\frac{1}{3}}},
$$

where $\xi, m^{*}, q$, and $\hbar$ are the electric field, effective mass, elementary charge, and Dirac's constant, respectively.

To experimentally elucidate the Franz-Keldysh effect, we conducted spectroscopic measurements on the GaN samples in the electrolyte under $V_{\mathrm{a}}$. Figure 4 a shows the increases in photo-absorbance measured as a function of the wavelength of incident light, where the vertical axis shows the difference between the data obtained at $V_{\mathrm{a}}=$ $3 \mathrm{~V}$ and $V_{\mathrm{a}}=0 \mathrm{~V}$. The data clearly show that the red-shift of the absorption edge under $V_{\mathrm{a}}=3 \mathrm{~V}$ was applied. The highest increase in absorbance was obtained at a wavelength of $370 \mathrm{~nm}$, corresponding to the largest anodic-reaction current density obtained, as shown in Table I. The absorbance $A\left(h \nu, V_{\mathrm{a}}\right)$ is expressed as

$$
A\left(h \nu, V_{a}\right)=1-\exp \left(-\alpha\left(h \nu, V_{a}\right) W\left(V_{a}\right)\right),
$$

where $W$ is the depletion layer width obtained by solving the Poisson's equation in the depletion region. The solid line in Fig. 4a is the theoretical curve of the increase in absorbance obtained from Eq. 4 by considering the Franz-Keldysh effect expressed with Eqs. 2 


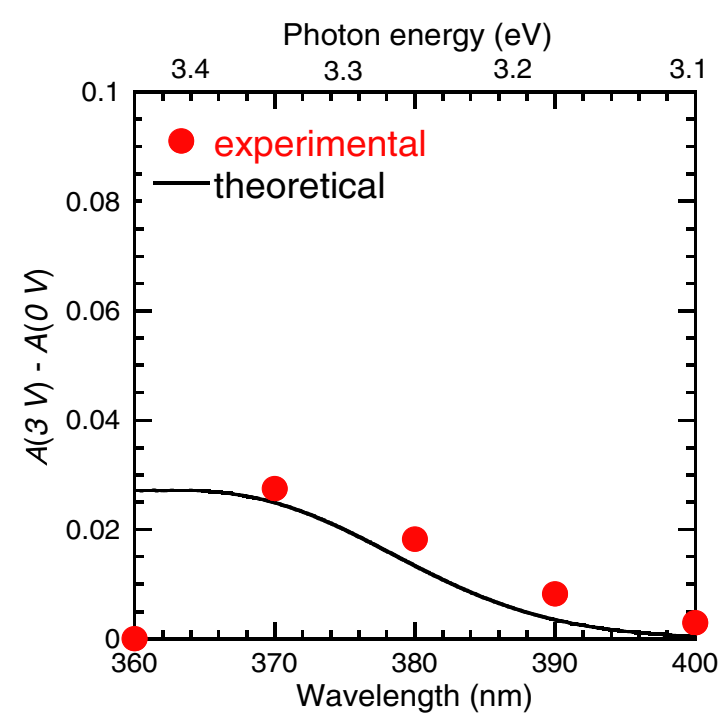

(a)

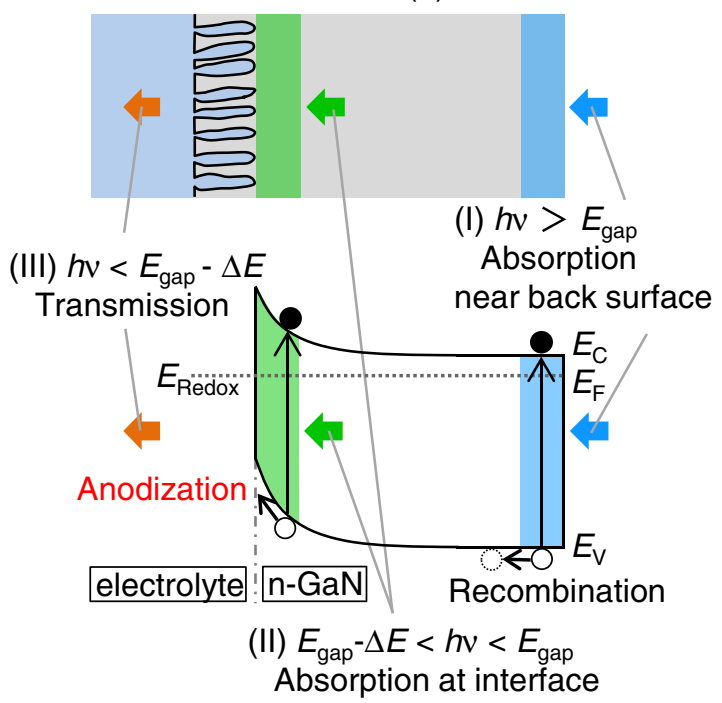

(b)

Figure 4. (a) Photo-absorbance measured as function of wavelength of incident light and (b) formation model of GaN-porous structure using photoassisted electrochemical process.

and 3. As shown in Fig. 4a, we found that the experimental data were well reproduced by the theoretical curve using the reasonable value of $\alpha_{0}=18000 \mathrm{~cm}^{-1}$ at $3.44 \mathrm{eV}$ for $\mathrm{GaN} .{ }^{19}$ These results strongly suggest that the red-shift of the absorption edge observed for the formation of $\mathrm{GaN}$-porous structures arises from the Franz-Keldysh effect.

On the basis of the above discussion, we propose a formation model of GaN-porous structures in BSI mode. Figure $4 \mathrm{~b}$ shows our model with a schematic of a porous structure and its band-diagram during the photo-assisted electrochemical process. In the case of illumination with photon energy above the bulk bandgap $h \nu>E_{\text {gap }}$ the holes were generated near the back-surface due to so-called bandedge absorption (I). However, most of the holes were not involved in the anodic reaction since they recombined before reaching the reaction surface. For $E_{\text {gap }}-\Delta E<h v<E_{\text {gap }}$, the photons coming from the back-surface penetrated through the bulk GaN but were absorbed by Franz-Keldysh effect at the pore tips in which the high-electric field was induced under $V_{\mathrm{a}}$ (II). In such a situation, the holes are generated only at the pore tips and contribute to the anodization of $\mathrm{GaN}$ and pore formation. For $h v<E_{\text {gap }}-\Delta E$, the photons obviously penetrate thorough both the balk $\mathrm{GaN}$ and porous layer as transmitted light (III). Thus, the illumination with $h v$ between $E_{\text {gap }}-\Delta E$ and $E_{\text {gap }}$ plays the most important role in the formation of $\mathrm{GaN}$-porous structures in $\mathrm{BSI}$ mode. This model explains very consistently the experimental results obtained in BSI mode in which the pores were etched only at the pore tips in the vertical direction.

In summary, we succeeded in forming GaN-porous structures by using photo-assisted electrochemical etching. The morphology of GaN-porous structures depends on the direction of light illumination. We experimentally demonstrated that the pore depth linearly increased with $Q$ in the BSI mode because the pores were etched only at the pore tips. Our proposed model by considering the FranzKeldysh effect makes it possible to explain the experimental results consistently. From both the experimental results and theoretical analysis, the BSI mode is powerful for controlling the structural properties of GaN-porous structures such as pore diameter and $d_{\mathrm{p}}$.

\section{Acknowledgments}

This work was supported in part by a Grant-in-Aid for Scientific Research (B) - 25289079 from Japan Society for the Promotion of Science (JSPS).

\section{References}

1. T. Nishida, H. Saito, and N. Kobayashi, Applied Physics Letters 79, 711 (2001).

2. T. Kokawa, T. Sato, H. Hasegawa, and T. Hashizume, Journal of Vacuum Science \& Technology B 24, 1972 (2006).

3. K. Fujii, T. K. Karasawa, and K. Ohkawa, Japanese Journal of Applied Physics 44, L543 (2005).

4. L. T. Canham, Applied Physics Letters 57, 1046 (1990).

5. P. Schmuki, D. J. Lockwood, H. J. Labbé, and J. W. Fraser, Applied Physics Letter 69, 1620 (1996)

6. Y. Kumazaki, T. Kudo, Z. Yatabe, and T. Sato, Applied Surface Science 279, 116 (2013).

7. T. Sato, N. Yoshizawa, and T. Hashizume, Thin Solid Films 518, 4399 (2010).

8. A. P. Vajpeyi, S. J. Chua, S. Tripathy, and E. A. Fitzgerald, Applied Physics Letters 91, 083110 (2007).

9. H. Hartono, C. B. Soh, S. J. Chua, and E. A. Fitzgerald, Journal of the Electrochemical society 154, H1004 (2007)

10. A. Ramizy, Z. Hassan, and K. Omar, Sensors and Actuators B, 155699 (2011).

11. Y. Kumazaki, A. Watanabe, Z. Yatabe, and T. Sato, Journal of the Electrochemical Society 161, H705 (2014).

12. K. Al-Heuseen, M. R. Hashim, and N. K. Ali, Applied Surface Science, 257, 6197 (2011).

13. V. Lehmann and H. Föll, Journal of the Electrochemical Society 137, 653 (1990).

14. W. Franz, Z. Naturforschung 13a, 484 (1958).

15. L. V. Keldysh, Soviet Physics, JETP 7, 788 (1958).

16. A. Cavallini, L. Polenta, M. Rossi, T. Stoica, R. Calarco, R. J. Meijers, T. Richter, and H. Lüth, Nano letters 7, 2166 (2007).

17. H. Haug and W. KochS, Quantum Theory of the Optical and Electronic Properties of Semiconductors, World Scientific Publishing, p 343 (1994)

18. H. Y. Peng, M. D. McCluskey, Y. M. Gupta, M. Kneissl, and N. M. Johnson, Applied Physics Letters 82, 2085 (1989).

19. J. F. Muth, J. H. Lee, I. K. Shmagin, R. M Kolbas, H. C. Casey Jr. B. P. Keller, U. K. Mishra, and S. P. Denbaars, Applied Physics Letters 71, 2572 (1997). 